\title{
Topiramate: Safety in migraine patients
}

Sir,

Topiramate, a sulfa-derivative monosaccharide was originally developed as a hypoglycemic agent, but was found to be devoid of hypoglycemic activity, and later, it was approved in 1995 in the UK as adjunctive treatment for partial-onset seizures. ${ }^{1}$ Topiramate with several mechanisms of actions, by its long half-life, high oral bioavailability, low protein binding, renal elimination, and with little or no hepatic metabolism, proves to be an ideal migraine prophylaxis medication, and was approved in 2004 by the Food and Drug Administration. 2,3

Safety and efficacy of topiramate in the prophylactic treatment of migraine is well documented and it is evident that topiramate might be the therapeutic choice in patients not responding to classical first-line prophylactic treatment. ${ }^{4}$ The increase in the horizon of topiramate use, leads to increase in the range of its adverse effects. Notable adverse effects are dizziness, slowed thinking, somnolence, ataxia, fatigue, confusion, weight loss, impaired concentration, and paresthesias. ${ }^{5}$ The ophthalmological effect of topiramate, paresthesias, and itching were considered to be the treatment-emergent adverse reactions of topiramate. ${ }^{6}$

This article aims to categorize the various adverse effects of migraine patients treated with topiramate.

This prospective-observational study was conducted in the Department of Neurology and Department of Ophthalmology, Kovai Medical Center and Hospital, a multi-specialty hospital at Coimbatore, in Tamil Nadu, India for a period of 8 months from June 2010 to January 2011. This study was approved by the Institutional Ethics Committee (Kovai Medical Center and Hospital), Coimbatore, India (Ref.: EC/AP/136/06/2010).

Table 1

Patients demographic details $(n=31)$.

\begin{tabular}{ll}
\hline Patient's demographics & No. of patients $(n=31)$ \\
\hline Mean (SD) age of the study population & $27.45(4.21)$ \\
Number of female patients & 29 \\
Number of male patients & 2 \\
Educational qualification & \\
<10th standard & 6 \\
>10th standard and $\leq 12$ th standard & 14 \\
Graduates & 5 \\
Post graduates & 6 \\
Nature of work & \\
Home maker & 23 \\
Lecturers/teachers & 6 \\
Lorry driver & 1 \\
Business & 1 \\
Mean (SD) BMI of the study population & $29.26(3.54)$ \\
\hline
\end{tabular}

BMI: body mass index, SD: standard deviation.
The study was concerned with the patients diagnosed with migraine according to the International Headache Society criteria. The treatment was initiated to the patient with a dose of $50 \mathrm{mg} /$ day for a period of 10 days, and the dose was titrated to $100 \mathrm{mg} /$ day for a period of 6 months. The adverse effects experienced by the patient's owing to the therapy were monitored every month. The rise in intra-ocular pressure (IOP) was given crucial importance and monitored using Schiotz tonometer. The paired ' $t$ '-test was used for statistical analysis using SPSS (V-11.0). Values of $P \leq 0.05$ were taken to be significant.

In this study, the safety and tolerability of the medication was assessed among the 31 (29 female and 2 male) patients who were diagnosed as migraine for the first time. The mean age of the study population was found to be $27.45 \pm 4.2$. Of the study population, 11 patients were diagnosed as migraine with aura and 20 patients as migraine without aura. All the patients included in the study were literate and their demographics are illustrated in Table 1 .

The study patients' weight was monitored at the initial visit and their body mass index (BMI) was calculated and the average BMI was found to be 29.26 and hence were prescribed with topiramate, as this drug has been studied in obesity to induce weight reduction. ${ }^{7}$ Among the diverse adverse drug reactions of the drug, the effects described by the patients were dizziness, somnolence, and weight loss. Among the study population $(n=31), 25$ patients reported the adverse drug reaction, of which 10 patients reported weight loss, somnolence, and dizziness were reported by 5 and 6

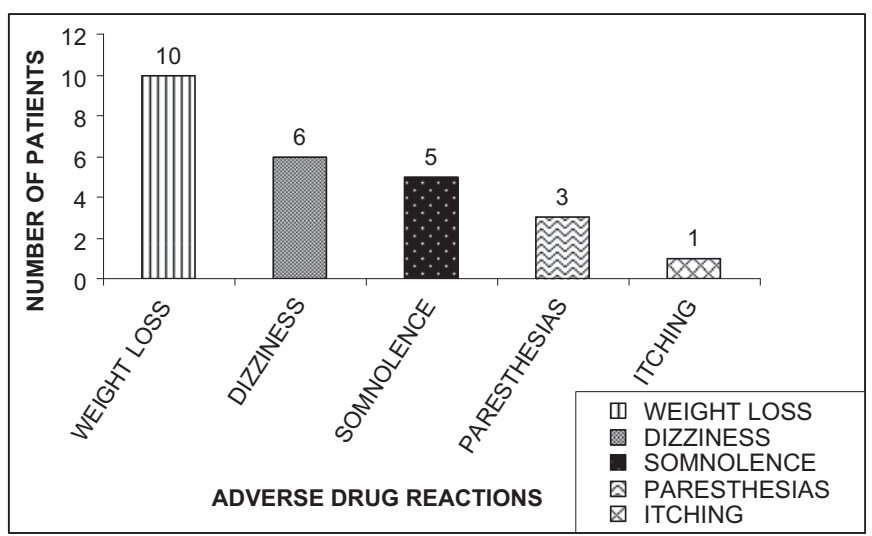

Fig. 1. Illustrates the number $(n=25)$ of patients experiencing adverse drug reactions. $X$-axis shows the various adverse drug reactions experienced by the patients. $Y$-axis shows the number of patients reported the adverse drug reactions. Weight loss $(n=10)$, dizziness $(n=6)$, somnolence $(n=5)$, paresthesias $(n=3)$, itching $(n=1)$. 


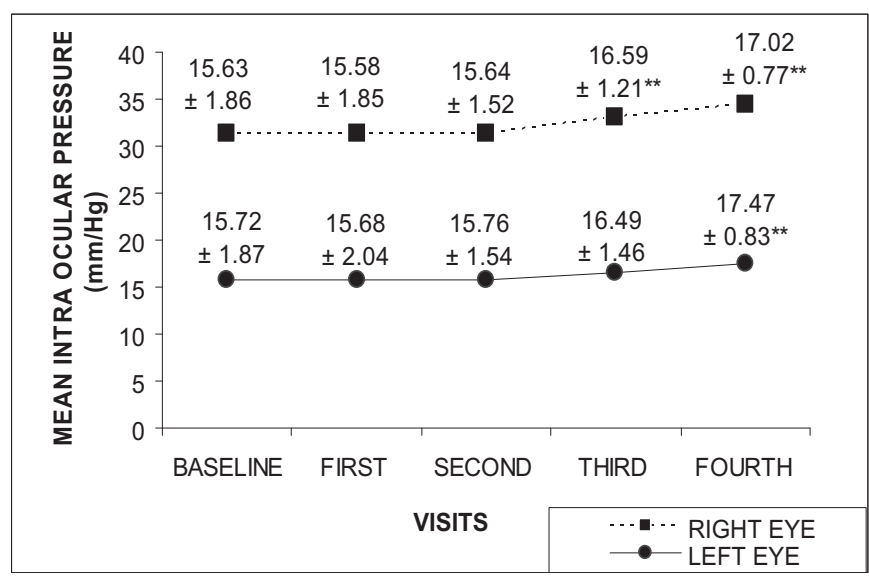

Fig. 2. Illustrates the mean (SD) intra-ocular pressure (IOP) of the patients at each visit. $X$-axis shows the visits and $Y$-axis shows the mean (SD) IOP. $\bullet$ - Left eye, $\mathbf{\square}$ - right eye. ${ }^{* *} P \leq 0.01$.

patients, respectively. Four patients in the study group reported paresthesias $(n=3)$ and itching $(n=1)$ and were discontinued from the treatment, and started with conventional therapy (Fig. 1). Six patients did not report any adverse drug reaction and responded well to the treatment.

The IOP of the patient was monitored bilaterally at each visit and it was noted that there was a significant $(P \leq 0.01)$ increase in the IOP from $15.72 \pm 1.87$ (right eye) and $15.63 \pm 1.86$ (left eye) at baseline to $17.47 \pm 0.83$ (right eye) and $17.02 \pm 0.77$ in the left eye at final visit (Fig. 2).

This study categorized the various adverse effects of topiramate in migraine patients. Further studies are warranted to evaluate the safety of topiramate for prophylaxis of migraine in patients' refractory to multiple therapies.

In conclusion, topiramate, an antiepileptic drug used in the prophylactic management of migraine though appears to contribute to the reduction in migraine frequency, also produces various treatment-emergent adverse drug reactions, which need to be given supreme importance. Neurologists, ophthalmologists, and clinical pharmacists should work collectively in improving the quality of life of patients.

\section{References}

1. Young WB, Hopkins MM, Shechter AL, Silberstein SD. Topiramate: a case series study in migraine prophylaxis. Cephalalgia. 2002;22:659-663.

2. White HS. Molecular pharmacology of topiramate: managing seizures and preventing migraine. Headache. 2005;45:S48-S56.

3. Sun C, Rapoport A. New treatment strategies for migraine prevention. A report. US Neurol Dis. 2006:18-22.

4. Silberstein SD, Lipton RB, Dodick DW, et al. Efficacy and safety of topiramate for the treatment of chronic migraine: a randomized, double-blind, placebo-controlled trial. Headache. 2007;47:170-180.

5. Diener HC, Bussone G, Van Oene JC, et al. Topiramate reduces headache days in chronic migraine: a randomized, double-blind, placebo-controlled study. Cephalalgia. 2007;27:814-823.

6. Abtahi MA, Abtahi SH, Fazel F, et al. Topiramate and the vision: a systematic review. Clin Ophthalmol. 2012;6:117-131.

7. Alberici A, Borroni B, Manelli F, et al. Topiramate weight loss in migraine patients. J Neurol Sci. 2009;278:64-65.

M. Ahamada Safna Mariyam* Department of Pharmacy Practice, Nandha College of Pharmacy, Erode, India

Suchandra Sen, K. Vijayan

Department of Pharmacy Practice, Drug and Poison Information Center, KMCH College of Pharmacy, Coimbatore, India

Department of Neurology, Kovai Medical Center and Hospital, Coimbatore, India

* Corresponding author. E-mail address: Safna86@gmail.com (M. Ahamada Safna Mariyam)

8 August 2012 Available online 6 March 2013 